\title{
Acidente vascular cerebral: um olhar para o perfil do cuidador
}

\author{
Stroke: a look at the profile of the caregiver \\ Accidente cerebrovascular: una mirada al perfil del cuidador
}

Kézia Porto Lima ${ }^{1 *}$, Beatriz Santos Araújo Silva ${ }^{1}$, Karla Katariny Nitão Loureiro Benedito Gomes ${ }^{1}$, Monica Cristina de Souza ${ }^{2}$, Cilene Aparecida de Souza Melo ${ }^{1,3}$, Sarah Lais Rocha ${ }^{3}$, Michele Pereira da Trindade Viera ${ }^{1,4}$.

\section{RESUMO}

Objetivo: Identificar o perfil do cuidador informal de pacientes com Acidente Vascular Cerebral (AVC) descritos na literatura científica e como se dá a designação desse papel. Métodos: Trata-se de uma revisão integrativa da literatura baseada em artigos indexados nas bases de dados LILACS e SCIELO, publicados no período de 2014 a 2019, disponíveis nos idiomas inglês, espanhol e português. Resultados: Foram selecionados 18 artigos científicos que responderam à pergunta de pesquisa, os achados apontam que a mulher é a principal cuidadora informal dos pacientes que sofreram um acidente vascular cerebral, possuem idade média de 55 anos; o nível de parentesco mais comum são os cônjuges e filhos; dedicam-se cerca de 20 horas por dia ao cuidado do familiar com sequelas pós AVC; apresentam pouca formação escolar e baixa renda financeira. Considerações Finais: $O$ conhecimento das características dos cuidadores informais pode orientar os profissionais de saúde a traçar cuidados mais direcionados de acordo com cada família; é imprescindível que haja suporte emocional e financeiro aos cuidadores.

Palavras-chave: Acidente vascular cerebral, Cuidados domiciliares de saúde, Cuidadores.

\begin{abstract}
Objective: To identify the profile of the informal caregiver of patients with Stroke described in the scientific literature and how this role is designated. Methods: This is an integrative literature review based on articles indexed in the LILACS and SCIELO databases, published between 2014 and 2019, available in English, Spanish and Portuguese. Results: 18 scientific articles were selected that answered the research question, the findings indicate that the woman is the main informal caregiver of patients who suffered a stroke, have an average age of 55 years; the most common level of kinship is spouses and children; they dedicate about 20 hours a day to the care of the family with sequelae after stroke; have little schooling and low financial income. Final Considerations: Knowledge of the characteristics of informal caregivers can guide health professionals to design more targeted care according to each family; it is essential that there is emotional and financial support for caregivers.
\end{abstract}

Keywords: Stroke, Home health care, Caregivers.

\section{RESUMEN}

Objetivo: Identificar el perfil del cuidador informal de pacientes con Ictus descrito en la literatura científica y cómo se designa este rol. Métodos: Se trata de una revisión de literatura integradora basada en artículos indexados en las bases de datos LILACS y SCIELO, publicados entre 2014 y 2019, disponible en inglés, español y portugués. Resultados: Se seleccionaron 18 artículos científicos que respondieron la pregunta de investigación, los hallazgos indican que la mujer es la principal cuidadora informal de los pacientes que sufrieron un ictus, tienen una edad promedio de 55 años; el nivel más común de parentesco son los cónyuges e hijos; dedican unas 20 horas diarias al cuidado de la familia con secuelas tras el ictus; tienen poca escolaridad y bajos ingresos económicos. Consideraciones Finales: El conocimiento de las características de los cuidadores informales puede orientar a los profesionales de la salud a diseñar cuidados más focalizados de acuerdo a cada familia; es fundamental que exista apoyo emocional y económico para los cuidadores.

Palabras clave: Ictus, Atención domiciliaria, Cuidadores.

\footnotetext{
${ }^{1}$ Faculdade Carajás, Marabá - PA. *E-mail: keziaporto@hotmail.com

${ }^{2}$ Faculty of Medical Sciences, State University, Rio de Janeiro (FCM/UERJ), Rio de Janeiro - RJ.

${ }^{3}$ Universidade do Estado do Pará (UEPA), Marabá - PA.

${ }^{4}$ Faculdade de Medicina de Marabá (FACIMPA), Marabá - PA.
} 


\section{INTRODUÇÃO}

O rápido envelhecimento populacional no mundo é um dos aspectos mais importantes e dinâmicos da demografia moderna, trazendo consigo desafios para os sistemas de saúde. A partir disso, evidencia-se uma transição epidemiológica que representa mudança no padrão de morbimortalidade, implicando em uma nova tendência: o aumento do número de casos de doenças crônico-degenerativas em relação às enfermidades infecciosas e parasitárias (ARAUJO JB, et al., 2016).

Para a Organização Pan-Americana da Saúde (OPAS), as doenças cardiovasculares são a principal causa de morte no mundo, sendo o Acidente Vascular Cerebral (AVC) e a cardiopatia isquêmica responsáveis por cerca de 15,2 milhões de óbitos em 2016 (OPAS, 2016).

Nesse contexto, o AVC é reportado como um problema de saúde de primeira ordem, caracterizado pela interrupção do fluxo sanguíneo cerebral; causando alterações histopatológicas e perda das habilidades cognitivas e motoras (CAMAK DJ, 2015; ESPUELA LF, et al., 2018).

De acordo com a Linha de Cuidados em AVC na Rede de Atenção às Urgências e Emergências do Sistema Único de Saúde (SUS), a mortalidade nos primeiros 30 dias após este evento neurológico é de 10\%, atingindo $40 \%$ no primeiro ano, e quando não é letal, o AVC causa incapacidade funcional e cognitiva em cerca de $45 \%$ dos sobreviventes, necessitando de reabilitação para essas consequências (Brasil, 2012).

A maioria dos sobreviventes apresentará sequelas neurológicas incapacitantes como as limitações motoras, sensitivas, sensoriais, de compreensão, expressão dos pensamentos, mudanças de personalidade e comportamentais; portanto, há restrição das Atividades de Vida Diárias (AVD) acarretando mudança no estilo de vida do paciente como impossibilidade de retorno ao trabalho, déficit na autonomia e na participação social (ARAUJO JB, et al., 2016).

No entanto, os avanços na reabilitação do AVC reduziram a incapacidade funcional grave e a longa permanência em instituições de cuidados em saúde, refletindo no aumento do número de pacientes que recebem assistência domiciliar de cuidadores informais (OLAI L, et al., 2015).

Nesse cenário, surge a figura do cuidador informal, o qual é definido como qualquer parente, amigo ou vizinho que tenha um relacionamento pessoal significativo e forneça uma ampla assistência de maneira altruísta e voluntária a pessoas com uma condição crônica ou incapacitante (KRIEGER T, et al., 2016); de acordo com Araújo JB, et al. (2016) o grau de incapacidade do paciente indica o quanto este será dependente do cuidador.

As limitações funcionais, principalmente na fase crônica do AVC, trazem repercussões também na esfera social da vida dos cuidadores como modificações nas relações familiares, dificuldade de se manter no trabalho e sentimento de desesperança em relação ao futuro; além disso, a prestação de cuidados representa custos físicos, sociais e financeiros. Essa sobrecarga torna as vivências familiares esgotantes ao se depararem com esse conjunto de transformações complexas e desafios diários que surgem mediante a aplicação dos cuidados ao doente (COSTA TF, et al., 2016).

Dessa forma, Arruda GO, et al. (2018) pontua que o desempenho do papel de cuidador informal constitui uma tarefa desgastante, o que representa um potencial fator de risco para desordens físicas e psíquicas. Assim, a família do paciente sofre frequentes rearranjos para prestar o cuidado, entendidos como um conjunto de movimentos realizados pelos familiares para atender às demandas e necessidades da pessoa adoecida envolvendo-se totalmente às suas demandas (COSTA TF, et al., 2016; ARRUDA GO, et al., 2018).

Para Silva JK, et al. (2016) é imprescindível conhecer as características dos cuidadores informais, de modo a contribuir com a equipe multiprofissional na elaboração dos planos de cuidados domiciliares, para que assim se consolide as estratégias de políticas públicas que os inserem na rede assistencial de saúde, viabilizando uma assistência em saúde melhor direcionada.

Nesse sentido, o presente estudo buscou identificar o perfil do cuidador informal de pacientes com AVC descritos na literatura científica, bem como compreender como ocorre a escolha desse papel e os principais cuidados empregados por ele. 


\section{MÉTODOS}

Trata-se de uma Revisão Integrativa da Literatura (RIL) que busca reunir informações relacionadas ao perfil do cuidador informal de pacientes após a ocorrência do AVC. Foi realizada buscas em bases de dados científicas em outubro de 2019, utilizando o formulário de busca avançada nas seguintes bases de dados: Literatura Latino-Americana e do Caribe em Ciências da Saúde (LILACS) e Scientific Eletronic Library Online (SciELO), mediante o emprego dos Descritores em Ciências da Saúde (DECS): "Acidente Vascular Cerebral", "Cuidados domiciliares de saúde" e "Cuidadores".

Os critérios de inclusão definidos para a seleção dos artigos foram: artigos publicados nos idiomas português, espanhol e inglês que respondessem à questão norteadora deste estudo presentes nas bases de dados selecionadas publicados nos últimos 5 anos (2014 a 2019); foram excluídas dissertações, teses, produções repetidas (entre as bases de dados) e que não abordassem a temática do estudo. A inserção dos artigos está melhor apresentada na Tabela 1.

Tabela 1 - Critérios de inclusão e exclusão de acordo com as bases de dados selecionadas.

\begin{tabular}{lccc}
\hline Produção & LILACS & SCIELO & Total \\
\hline Produção encontrada & 67 & 8 & 75 \\
Não publicado em português, inglês ou espanhol & 4 & - & 4 \\
Não abordam a temática em estudo & 42 & - & 42 \\
Não foi publicado nos últimos 5 anos & - & 7 & 7 \\
Dissertações ou teses & 3 & - & 3 \\
Repetidos & Total & 1 & - \\
\hline
\end{tabular}

Fonte: Lima KP, et al., 2020

\section{RESULTADOS E DISCUSSÂO}

Foram encontrados 75 artigos utilizando-se os critérios de busca previamente definidos, após a leitura de títulos e resumos, selecionou-se 17 artigos na base LILACS e 1 na SciELO.

Evidenciou-se que o maior número de produções científicas sobre o perfil do cuidador informal de paciente com AVC foi publicado pelo Reino Unido, equivalendo 33,3\% do total, seguido por estudos dos Estados Unidos (27,7\%) e Brasil (22,2\%). A Espanha, o Canadá e a Suécia corresponderam igualmente a 5,6\% dentre as publicações.

Quanto à metodologia, os artigos apresentavam-se em diversos métodos científicos com predominância de estudos transversais $(55,5 \%)$ e publicados em diferentes periódicos. Destaca-se que a maioria dos estudos foram produzidos por profissionais enfermeiros $(72,2 \%)$, terapeutas ocupacionais (16,7\%) e médicos $(11,1 \%)$. $\mathrm{O}$ ano de 2015 deteve o maior número de publicações (33,3\%). Os 18 artigos que constituíram a amostra do estudo e as suas características estão representadas no Quadro 1. 
Revista Eletrônica Acervo Saúde / Electronic Journal Collection Health | ISSN 2178-2091

Quadro 1 - Representação da síntese da produção científica analisada.

\begin{tabular}{|c|c|c|c|c|c|c|c|}
\hline Base & Título & Autores & País & Ano & $\begin{array}{l}\text { Método (tipo } \\
\text { de estudo) }\end{array}$ & Objetivo & Resultados \\
\hline LILACS & $\begin{array}{c}\text { Critical points in the } \\
\text { experience of spouse } \\
\text { caregivers of patients } \\
\text { who have suffered a } \\
\text { stroke. A } \\
\text { phenomenological } \\
\text { interpretive study }\end{array}$ & Espuela LF, et al. & EUA & 2018 & $\begin{array}{c}\text { Estudo } \\
\text { fenomenológi } \\
\text { co qualitativo. }\end{array}$ & $\begin{array}{l}\text { Explorar e documentar as } \\
\text { experiências e os valores dos } \\
\text { cuidadores de cônjuges de } \\
\text { sobreviventes de AVC. }\end{array}$ & $\begin{array}{l}\text { Cuidadores de cônjuges sobreviventes de AVC } \\
\text { passam por um processo de interrupção em suas } \\
\text { vidas e relacionamentos particulares, marcado por } \\
\text { seus deveres de cuidar. }\end{array}$ \\
\hline LILACS & $\begin{array}{l}\text { Percepções da família } \\
\text { acerca do cuidado ao } \\
\text { homem com alguma } \\
\text { condição crônica }\end{array}$ & Arruda GO, et al. & Brasil & 2018 & $\begin{array}{l}\text { Transversal, } \\
\text { descritivo, } \\
\text { exploratório }\end{array}$ & $\begin{array}{l}\text { Conhecer as percepções da } \\
\text { família acerca do cuidado a } \\
\text { homens com condições } \\
\text { crônicas e dependentes de } \\
\text { cuidados domiciliares. }\end{array}$ & $\begin{array}{l}\text { A maior parte dos familiares cuidadores eram do } \\
\text { sexo feminino, tinha idade entre } 49 \text { e } 83 \text { anos e } \\
\text { apresentava alguma condição crônica. Os } \\
\text { cuidados referidos foram desde o auxílio no banho } \\
\text { até o manejo de dispositivos mais complexos. }\end{array}$ \\
\hline LILACS & $\begin{array}{l}\text { Perfil de cuidadores } \\
\text { familiares de idosos } \\
\text { após o Acidente } \\
\text { Vascular Cerebral }\end{array}$ & Silva JK, et al. & Brasil & 2016 & $\begin{array}{l}\text { Corte } \\
\text { transversal, } \\
\text { descritivo e } \\
\text { exploratório }\end{array}$ & $\begin{array}{c}\text { Descrever o perfil dos } \\
\text { Cuidadores Familiares (CFs) de } \\
\text { idosos sobreviventes ao AVC e } \\
\text { o nível de sobrecarga de } \\
\text { cuidado. }\end{array}$ & $\begin{array}{c}\text { As CFs são mulheres (100\%), geralmente filhas e } \\
\text { esposas (76,9\%), com idade entre } 41 \text { e } 60 \text { anos } \\
(76,9 \%) \text {, baixa escolaridade }(64,3 \%) \text {, sem } \\
\text { atividade laboral }(84,6 \%) \text {, apresentam patologias } \\
(69,2 \%) \text { e sobrecarga moderada } \\
\text { de cuidado }(69,2 \%) .\end{array}$ \\
\hline LILACS & $\begin{array}{l}\text { Long-term impact of } \\
\text { stroke on family } \\
\text { caregiver well-being }\end{array}$ & Haley WE, et al. & EUA & 2015 & $\begin{array}{c}\text { Caso-controle } \\
\text { transversal }\end{array}$ & $\begin{array}{l}\text { Estudar mudanças de três anos } \\
\text { no bem-estar entre cuidadores } \\
\text { familiares de uma amostra } \\
\text { derivada de sobreviventes de } \\
\text { AVC e comparados com não } \\
\text { cuidadores correspondentes. }\end{array}$ & $\begin{array}{l}\text { Cuidadores que tinham escolaridade e renda } \\
\text { significativamente menor eram mais propensos a } \\
\text { relatar depressão, histórico de doença pulmonar } \\
\text { crônica, acidente vascular cerebral, dificuldade em } \\
\text { ver ou ouvir. }\end{array}$ \\
\hline
\end{tabular}

REAS / EJCH | Vol.12(11) | e4677 | DOI: https://doi.org/10.25248/reas.e4677.2020 Página 4 de 12 
Revista Eletrônica Acervo Saúde / Electronic Journal Collection Health | ISSN 2178-2091

\begin{tabular}{|c|c|c|c|c|c|c|c|}
\hline Base & Título & Autores & País & Ano & $\begin{array}{l}\text { Método (tipo } \\
\text { de estudo) }\end{array}$ & Objetivo & Resultados \\
\hline LILACS & $\begin{array}{l}\text { Addressing the burden } \\
\text { of stroke caregivers: a } \\
\text { literature review }\end{array}$ & Camak DJ & $\begin{array}{l}\text { Reino } \\
\text { Unido }\end{array}$ & 2015 & $\begin{array}{l}\text { Revisão de } \\
\text { literatura }\end{array}$ & $\begin{array}{l}\text { Examinar a literatura empírica } \\
\text { sobre o risco de sobrecarga } \\
\text { vivenciado pelo cuidador de } \\
\text { idosos do sobrevivente de } \\
\text { AVC. }\end{array}$ & $\begin{array}{l}\text { Frequentemente, o cuidador é o cônjuge do } \\
\text { sobrevivente do derrame que é empurrado para o } \\
\text { papel repentinamente e deve fornecer o cuidado } \\
\text { fisicamente extenuante. Assumir o papel de } \\
\text { cuidador tem um risco inerente que pode resultar } \\
\text { em comprometimentos de saúde para o cuidador. }\end{array}$ \\
\hline LILACS & $\begin{array}{l}\text { Independence and } \\
\text { cognition post-stroke } \\
\text { and its } \\
\text { relationship to burden } \\
\text { and quality of life of } \\
\text { family } \\
\text { caregivers }\end{array}$ & Caro CC, et al. & $\begin{array}{l}\text { Reino } \\
\text { Unido }\end{array}$ & 2016 & $\begin{array}{l}\text { Transversal e } \\
\text { correlacional }\end{array}$ & $\begin{array}{c}\text { Investigar se existe uma } \\
\text { correlação entre os níveis de } \\
\text { independência e cognição em } \\
\text { pacientes com AVC e a } \\
\text { qualidade de vida de seus } \\
\text { cuidadores. }\end{array}$ & $\begin{array}{l}\text { Há uma forte correlação positiva entre } \\
\text { independência e cognição e uma moderada } \\
\text { correlação negativa entre independência e } \\
\text { qualidade de vida dos cuidadores. A amostra de } \\
\text { cuidadores incluiu predominantemente adultos } \\
\text { mulheres, casadas e também com baixos níveis } \\
\text { de educação escolar. }\end{array}$ \\
\hline LILACS & $\begin{array}{l}\text { To be or not to be? A } \\
\text { caregiver's question: } \\
\text { the lived experience of } \\
\text { a stroke family during } \\
\text { the first } 18 \text { months } \\
\text { poststroke }\end{array}$ & Olivier CL, et al. & $\begin{array}{l}\text { Reino } \\
\text { Unido }\end{array}$ & 2017 & $\begin{array}{c}\text { Fenomenológi } \\
\text { co }\end{array}$ & $\begin{array}{c}\text { Exploraras experiência de três } \\
\text { familiares de AVC durante } 18 \\
\text { meses após um primeiro } \\
\text { derrame. }\end{array}$ & $\begin{array}{l}\text { A amostra incluiu predominantemente esposas; os } \\
\text { profissionais precisam considerar o impacto do } \\
\text { cuidado prolongado ao familiar. }\end{array}$ \\
\hline LILACS & $\begin{array}{c}\text { Impacto en la calidad } \\
\text { de vida en cuidadores } \\
\text { de supervivientes de un } \\
\text { ictus }\end{array}$ & Espuela LF, et al. & Espanha & 2015 & $\begin{array}{l}\text { Descritivo e } \\
\text { transversal }\end{array}$ & $\begin{array}{l}\text { Determinar a qualidade de vida } \\
\text { relacionada à saúde e o grau } \\
\text { de sobrecarga do cuidador de } \\
\text { um paciente após um acidente } \\
\text { vascular cerebral. }\end{array}$ & $\begin{array}{l}58 \% \text { relataram ter humor deprimido, } 31,3 \% \\
\text { sobrecarga e } 89,6 \% \text { problemas de sono. A idade } \\
\text { média foi de } 55,63 \text { e a maioria eram mulheres } \\
(70,8 \%) \text { e o número médio de horas que eles se } \\
\text { dedicaram ao cuidar de seu parente era } 21,71 \text {. O } \\
\text { relacionamento mais frequente na amostra foi } \\
\text { cônjuge }(66,7 \%) \text {. A maioria das famílias tinha } \\
\text { renda entre } 500 \text { e } 1.000 \text { euros. }\end{array}$ \\
\hline
\end{tabular}

REAS / EJCH | Vol.12(11) | e4677 | DOI: https://doi.org/10.25248/reas.e4677.2020 Página 5 de 12 
Revista Eletrônica Acervo Saúde / Electronic Journal Collection Health | ISSN 2178-2091

\begin{tabular}{|c|c|c|c|c|c|c|c|}
\hline Base & Título & Autores & País & Ano & $\begin{array}{c}\text { Método (tipo } \\
\text { de estudo) }\end{array}$ & Objetivo & Resultados \\
\hline LILACS & $\begin{array}{c}\text { Health education for } \\
\text { stroke patient carers: } \\
\text { Does it affect functional } \\
\text { status improvement in } \\
\text { patients after ischemic } \\
\text { stroke? }\end{array}$ & Hebel K, et al. & EUA & 2014 & $\begin{array}{c}\text { Coorte } \\
\text { prospectivo }\end{array}$ & $\begin{array}{l}\text { Avaliar o impacto do } \\
\text { treinamento de cuidadores } \\
\text { melhora do estado funcional } \\
\text { em pacientes após AVC. }\end{array}$ & $\begin{array}{c}\text { O status funcional dos pacientes de acordo com o } \\
\text { Índice de Barthel e a Escala de Rankin Modificada } \\
\text { melhorou significativamente em ambos os grupos. } \\
\text { No grupo controle, a maioria dos cuidadores eram } \\
\text { cônjuges dos pacientes (59\%), filhos ou parentes } \\
\text { (38\%). }\end{array}$ \\
\hline LILACS & $\begin{array}{l}\text { Developing a complex } \\
\text { intervention programme } \\
\text { for informal } \\
\text { caregivers of stroke } \\
\text { survivors: The } \\
\text { Caregivers' Guide }\end{array}$ & Krieger $\mathrm{T}$, et al. & $\begin{array}{l}\text { Reino } \\
\text { Unido }\end{array}$ & 2016 & $\begin{array}{l}\text { Qualitativo } \\
\text { exploratório }\end{array}$ & $\begin{array}{l}\text { Desenvolver um programa para } \\
\text { cuidadores de AVC na Renânia } \\
\text { do Norte-Vestfália, Alemanha. }\end{array}$ & $\begin{array}{l}\text { Os cuidadores tinham idade entre } 47 \text { e } 78 \text { anos } \\
\text { (mediana } 62 \text { anos) e estavam relacionados ao } \\
\text { sobrevivente do AVC como parceiro ou filho } \\
\text { adulto. } 6 \text { participantes eram do sexo feminino e } 5 \\
\text { estavam aposentados. O programa de suporte } \\
\text { recém-desenvolvido consiste em cinco blocos: } \\
\text { Conteúdo Humano, Recursos, abordagem } \\
\text { personalizada, tempo e configuração. }\end{array}$ \\
\hline LILACS & $\begin{array}{l}\text { A home-based training } \\
\text { programme } \\
\text { improves family } \\
\text { caregivers' oral care } \\
\text { practices with stroke } \\
\text { survivors:a randomized } \\
\text { controlled trial }\end{array}$ & Kuo YW, et al. & $\begin{array}{l}\text { Reino } \\
\text { unido }\end{array}$ & 2015 & Randomizado & $\begin{array}{l}\text { Avaliar a eficácia de um } \\
\text { programa de tratamento bucal } \\
\text { domiciliar. }\end{array}$ & $\begin{array}{c}\text { Na amostra, } 31,3 \% \text { eram cônjuges, } 52,1 \% \text { eram } \\
\text { filhos, e } 16,7 \% \text { tinha outro parentes. } 46 \% \text { dos } \\
\text { cuidadores familiares eram mulheres e idade } \\
\text { média de } 53,9 \text { anos. As evidências demonstraram } \\
\text { que o grupo de intervenção detinham mais } \\
\text { conhecimento maior e melhor comportamento de } \\
\text { higiene bucal. }\end{array}$ \\
\hline LILACS & $\begin{array}{l}\text { Burden and Quality of } \\
\text { Life of Family } \\
\text { Caregivers of Stroke } \\
\text { Patients }\end{array}$ & Caro CC, et al. & Canadá & 2018 & $\begin{array}{l}\text { Descritivo, } \\
\text { transversal }\end{array}$ & $\begin{array}{l}\text { Identificar o nível de carga e } \\
\text { qualidade de vida de } \\
\text { cuidadores familiares de } \\
\text { pacientes com AVC. }\end{array}$ & $\begin{array}{l}\text { Cuidadores de pacientes com AVC apresentaram } \\
\text { níveis moderados de carga e redução na } \\
\text { qualidade da vida. Os cuidadores eram } \\
\text { principalmente mulheres ( } 90 \% \text { ), casados ( } 83 \%) \text {, } \\
4,9 \text { anos de estudo, } 57 \% \text { eram esposas, } 23 \% \\
\text { eram filhas e } 7 \% \text { eram a mãe. }\end{array}$ \\
\hline
\end{tabular}

REAS / EJCH | Vol.12(11) | e4677 | DOI: https://doi.org/10.25248/reas.e4677.2020 Página 6 de 12 
Revista Eletrônica Acervo Saúde / Electronic Journal Collection Health | ISSN 2178-2091

\begin{tabular}{|c|c|c|c|c|c|c|c|}
\hline Base & Título & Autores & País & Ano & $\begin{array}{l}\text { Método (tipo } \\
\text { de estudo) }\end{array}$ & Objetivo & Resultados \\
\hline LILACS & $\begin{array}{l}\text { Task Difficulty and Life } \\
\text { Changes among Stroke } \\
\text { Family Caregivers: } \\
\text { Relationship to } \\
\text { Depressive Symptoms }\end{array}$ & $\begin{array}{l}\text { McLennon SM, et } \\
\text { al. }\end{array}$ & EUA & 2014 & $\begin{array}{c}\text { Transversal, } \\
\text { Descritivo }\end{array}$ & $\begin{array}{c}\text { Investigar diferenças na } \\
\text { dificuldade da tarefa do } \\
\text { cuidador de AVC e mudanças } \\
\text { na vida. }\end{array}$ & $\begin{array}{c}47 \% \text { dos cuiadores apresentavam sintomas leves } \\
\text { a graves de depressão e múltiplas condições de } \\
\text { saúde e limitações físicas. A maioria dos } \\
\text { cuidadores eram do sexo feminino, com idade } \\
\text { média de } 54,2 \text { anos. Havia um pouco mais de } \\
\text { cuidadores do cônjuge (52\%) do que cuidadores } \\
\text { do cônjuge (48\%). }\end{array}$ \\
\hline LILACS & $\begin{array}{l}\text { Life situations and the } \\
\text { care burden for stroke } \\
\text { patients and their } \\
\text { informal caregivers in a } \\
\text { prospective cohort } \\
\text { study }\end{array}$ & Olai L, et al. & Suécia & 2015 & Longitudinal & $\begin{array}{l}\text { Analisar se a situação de vida } \\
\text { paralela entre pacientes com } \\
\text { AVC e seus } \\
\text { cuidadores informais mostrados } \\
\text { em estudos transversais } \\
\text { prevalecem também em uma } \\
\text { perspectiva longitudinal. }\end{array}$ & $\begin{array}{c}\text { Os cuidadores informais estavam sob considerável } \\
\text { tensão e escores de ansiedade e depressão. Dois } \\
\text { terços dos cuidadores eram mulheres e idade } \\
\text { média foi de } 63 \text { anos, } 45 \% \text { foram cônjuges, } 40 \% \\
\text { eram filhos dos pacientes, } 48 \% \text { moravam na } \\
\text { mesma casa. }\end{array}$ \\
\hline LILACS & $\begin{array}{l}\text { The Effect of Cognitive } \\
\text { Appraisal on Quality } \\
\text { of Life of Providers of } \\
\text { Home Care for Patients } \\
\text { With Stroke }\end{array}$ & Pai HC e Tsay YC. & EUA & 2016 & $\begin{array}{l}\text { Transversal, } \\
\text { descritivo e } \\
\text { correlacional. }\end{array}$ & $\begin{array}{c}\text { Identificar se a avaliação } \\
\text { cognitiva influencia a qualidade } \\
\text { de vida relacionada à saúde. }\end{array}$ & $\begin{array}{l}\text { Os resultados indicaram que } 61 \% \text { dos } \\
\text { participantes avaliaram sua saúde como ruim ou } \\
\text { regular. A maioria dos cuidadores era do sexo } \\
\text { feminino }(58,4 \% \text { ) e familiares imediatos (pais, } \\
\text { cônjuge, filho, ou irmão; } 81,8 \% \text { e idade média = } \\
59,47 .\end{array}$ \\
\hline
\end{tabular}

REAS / EJCH | Vol.12(11) | e4677 | DOI: https://doi.org/10.25248/reas.e4677.2020 Página 7 de 12 
Revista Eletrônica Acervo Saúde / Electronic Journal Collection Health | ISSN 2178-2091

\begin{tabular}{|c|c|c|c|c|c|c|c|}
\hline Base & Título & Autores & País & Ano & $\begin{array}{l}\text { Método (tipo } \\
\text { de estudo) }\end{array}$ & Objetivo & Resultados \\
\hline LILACS & $\begin{array}{l}\text { The effect of cognitive } \\
\text { appraisal in middle- } \\
\text { aged women stroke } \\
\text { survivors and the } \\
\text { psychological health of } \\
\text { their caregivers: a } \\
\text { follow-up } \\
\text { study }\end{array}$ & Wu MH, et al. & $\begin{array}{l}\text { Reino } \\
\text { Unido }\end{array}$ & 2015 & Longitudinal & $\begin{array}{l}\text { Identificar os fatores que } \\
\text { afetam os aspectos } \\
\text { relacionados à saúde de } \\
\text { cuidadores e pacientes } \\
\text { mulheres, seis meses após o } \\
\text { AVC. }\end{array}$ & $\begin{array}{c}\text { Os determinantes da depressão dos cuidadores } \\
\text { eram o fardo; a média de idade dos cuidadores } \\
\text { era } 41,56 \text { anos; } 78 \% \text { tinham nível educacional } \\
\text { alto; a maioria eram filhas. }\end{array}$ \\
\hline LILACS & $\begin{array}{l}\text { A newly designed } \\
\text { intensive caregiver } \\
\text { education } \\
\text { program reduces } \\
\text { cognitive impairment, } \\
\text { anxiety, and } \\
\text { depression in patients } \\
\text { with acute ischemic } \\
\text { stroke }\end{array}$ & ZHANG L, et al. & Brasil & 2019 & $\begin{array}{l}\text { Caso-controle } \\
\text { transversal }\end{array}$ & $\begin{array}{l}\text { Avaliar o efeito de um novo } \\
\text { programa intensivo de } \\
\text { educação para cuidadores } \\
\text { (ICEP) na redução do } \\
\text { comprometimento cognitivo, } \\
\text { ansiedade e depressão em } \\
\text { pacientes com AVC isquêmico } \\
\text { agudo. }\end{array}$ & $\begin{array}{c}\text { O programa reduziu o comprometimento, } \\
\text { ansiedade e depressão em pacientes e os } \\
\text { cuidadores desempenham papéis importantes e } \\
\text { deven ser considerados pelo programa } \\
\text { educacional. A idade média dos cuidadores no O } \\
\text { grupo ICEP e o grupo Controle foram } 47,3 \text { e } 46,6 \text {, } \\
\text { respectivamente. A proporção de cuidadores } \\
\text { homem-mulher foi de } 30 / 68 \text { no grupo ICEP e } \\
32 / 66 \text { no grupo Controle. }\end{array}$ \\
\hline SciELO & $\begin{array}{c}\text { Acidente vascular } \\
\text { encefálico: } \\
\text { características do } \\
\text { paciente } \\
\text { e qualidade de vida de } \\
\text { cuidadores }\end{array}$ & Costa TF, et al. & Brasil & 2016 & $\begin{array}{l}\text { Descritiva, } \\
\text { transversal, } \\
\text { quantitativa }\end{array}$ & $\begin{array}{l}\text { Investigar a associação entre } \\
\text { os domínios da qualidade de } \\
\text { vida relacionada à saúde dos } \\
\text { cuidadores familiares e as } \\
\text { características } \\
\text { sociodemográficas dos } \\
\text { indivíduos com sequelas de } \\
\text { AVC. }\end{array}$ & $\begin{array}{l}\text { As menores médias dos escores na qualidade de } \\
\text { vida dos cuidadores foram das dimensões Dor, } \\
\text { Saúde mental e Aspectos sociais. Verificou-se que } \\
\text { a maioria era do sexo feminino ( } 84,50 \%) \text {, filhos } \\
\text { (51,47\%), idade média de } 34 \text { a } 47 \text { anos, casados } \\
\text { (57,35\%), com escolaridade entre cinco e nove } \\
\text { anos ( } 51,47 \%) \text {. Houve predominância de } \\
\text { cuidadores desempregados ( } 45,59 \%) \text {, com renda } \\
\text { pessoal entre um e três salários mínimos } \\
\text { (58,09\%). }\end{array}$ \\
\hline
\end{tabular}

Fonte: Lima KP, et al., 2020.

REAS / EJCH | Vol.12(11) | e4677 | DOI: https://doi.org/10.25248/reas.e4677.2020 Página 8 de 12 
A partir dos 18 artigos inseridos nesta RIL, foi possível traçar um esboço do perfil dos cuidadores informais de pacientes pós AVC: as mulheres são as principais cuidadoras informais em todos os estudos; com idade média de aproximadamente 55 anos; em relação ao grau de parentesco mais comum destaca-se os familiares imediatos como cônjuges e filhos(as); apresentam predominantemente baixa escolaridade; níveis reduzidos de empregos e baixa renda financeira; são casados ou estão em relacionamento estável e se dedicam cerca de 20 horas por dia ao cuidado do familiar (Quadro 2).

Quadro 2 - Características sociodemográficas de cuidadores informais segundo a literatura

\begin{tabular}{|c|c|c|c|}
\hline $\begin{array}{c}\text { Características dos } \\
\text { cuidadores }\end{array}$ & $\begin{array}{l}\text { № de } \\
\text { estudos }\end{array}$ & $\%$ & Autores \\
\hline Sexo feminino & 14 & 77,8 & $\begin{array}{l}\text { Silva JK, et al (2016); Arruda GO, et al (2018); Caro CC, et al (2016); Costa } \\
\text { TF, et al (2016); Kuo YW, et al (2016); Mclennon SM, et al (2014); Espuela } \\
\text { LF, et al (2015); Pai HC e Tsay YC (2016); Olai L, et al (2015); Olivier CL, } \\
\text { et al (2017); Krieger T, et al (2016); Wu MH, et al (2015); Zhang L, et al } \\
\text { (2019); Caro CC, et al (2018). }\end{array}$ \\
\hline$>55$ anos & 8 & 44,4 & $\begin{array}{l}\text { Pai HC e Tsay YC (2016); Kuo YW, et al (2016); Silva JK, et al (2016); } \\
\text { Mclennon SM, et al (2014); Olai L, et al (2015); Arruda GO, et al (2018); } \\
\text { Espuela LF, et al (2015); Krieger T, et al (2016). }\end{array}$ \\
\hline Cônjuges & 13 & 72,2 & $\begin{array}{l}\text { Hebel K, et al (2014); Kuo YW, et al (2016); Silva JK, et al (2016); } \\
\text { Mclennon SM, et al (2014); Espuela LF, et al (2015); Caro CC, et al (2016); } \\
\text { Krieger T, et al (2016); Espuela LF, et al (2018); Caro CC, et al (2018); Pai } \\
\text { HC e Tsay YC (2016); Olai L, et al (2015); Camak DJ (2015); Olivier CL, } \\
\text { et al (2017). }\end{array}$ \\
\hline Filhos(as) & 9 & 50 & $\begin{array}{l}\text { Costa TF, et al (2016); Hebel K, et al (2014); Wu MH, et al (2015); Silva } \\
\text { JK, et al (2016); Kuo YW, et al (2016); Krieger T, et al (2016); Caro CC, } \\
\text { et al (2018); Olai L, et al (2015; Pai HC e Tsay YC (2016); }\end{array}$ \\
\hline Baixa escolaridade & 7 & 38,9 & $\begin{array}{l}\text { Costa TF, et al (2016); Silva JK, et al (2016); Zhang-L, et al (2019); Caro } \\
\text { CC, et al (2016); Olai L, et al (2015); Haley WE, et al (2015); Caro CC, et } \\
\text { al (2018). }\end{array}$ \\
\hline $\begin{array}{l}\text { Baixa renda e } \\
\text { desempregados }\end{array}$ & 4 & 22,2 & $\begin{array}{l}\text { Costa TF, et al (2016); Caro CC, et al (2018); Silva JK, et al (2016); Espuela } \\
\text { LF, et al (2015). }\end{array}$ \\
\hline $\begin{array}{l}\text { Casados ou em } \\
\text { relacionamento } \\
\text { estável }\end{array}$ & 3 & 16,7 & Caro CC, et al (2016); Olai L, et al (2015); Haley WE, et al (2015). \\
\hline $\begin{array}{l}20 \text { horas dedicadas } \\
\text { ao cuidado }\end{array}$ & 2 & 11,1 & uela LF, et al (2015). \\
\hline
\end{tabular}

Fonte: Lima, KP, et al., 2020.

A experiência de sobreviver a um AVC é caracterizada por uma mudança radical na vida dos pacientes e de suas famílias, impactando as experiências físicas, psicológicas, sociais, emocionais e espirituais; as situações cotidianas realizadas com normalidade antes do AVC se tornam agora um verdadeiro desafio para os membros da família (PAI HC e TSAY YC, 2016; ESPUELA LF, et al., 2018; KRIEGER T, et al., 2016). Embora a doença possa afetar toda a família, somente um membro, geralmente, passa a ser o principal responsável pelo cuidado do paciente pós AVC. Dessa maneira, a designação do cuidador informal obedece a três fatores: 1. grau de parentesco, 2. gênero, 3. proximidade afetiva e física entre o paciente e o cuidador informal (CARO CC, et al., 2018).

Geralmente, o ato de cuidar, dentro do contexto familiar, é assumido pela mulher; afinal, do ponto de vista histórico, as atividades que ocorriam no ambiente domiciliar como cuidar da casa ou dos filhos sempre era atribuído a ela, enquanto o homem era responsável por garantir o provimento financeiro da família (SILVA JK, et al., 2016; ARRUDA GO, et al., 2018). Apesar de todas as mudanças sociais e dos novos papéis assumidos pela mulher no mercado de trabalho, ainda é comum que esta seja responsável pelo cuidado (CARO CC, et al., 2016).

O homem tem participado do cuidado de forma secundária por meio de ajuda financeira ou no transporte do paciente (COSTA TF et al., 2016). Assim, a cuidadora é encarregada por realizar diversas tarefas como cuidados de higiene pessoal, vestimenta, preparo e fornecimento da alimentação, além de ser responsável pelos afazeres domésticos; desse modo, a figura da mulher cuidadora não divergiu entre os países, sendo que essa característica cultural foi reconhecida e descrita em vários estudos (SILVA JK, et al., 2016; ARRUDA GO, et al., 2018; CARO CC, et al., 2016; COSTA TF, et al., 2016; KUO YW, et al., 2016; MCLENNON SM, et al., 2014; ESPUELA LF, et al., 2015; PAI HC e TSAY YC, 2016; OLAI L, et al., 2015; OLIVIER CL, et al., 2017; KRIEGER T, et al., 2016; WU MH, et al., 2015; ZHANG L, et al., 2019; CARO CC, et al., 2018). 
O parentesco tem influência decisiva na escolha do cuidador informal, visto que tem sido aquele que mantém o maior vínculo afetivo e o mais estreito grau de parentesco como cônjuges ou filhas, respectivamente (OLAI L, et al., 2015; PAI HC e TSAY YC, 2016). Segundo Olivier CL, et al. (2017), o cônjuge é movido pela obrigação matrimonial assumida pelo casamento e, portanto, depara-se com mudanças radicais e insatisfações conjugais.

No estudo de Espuela LF, et al. (2018) os entrevistados referiam que suas vidas estavam apenas centradas ao cuidado do familiar enfermo, e por isso, prevalecia o sentimento de desesperança em relação a seu futuro. Esse grau de parentesco compôs a maioria da amostra em diferentes estudos (HEBEL K, et al., 2014; KUO YW, et al., 2016; MCLENNON SM, et al., 2014; ESPUELA LF, et al., 2015; CARO CC, et al., 2016; PAI HC e TSAY YC, 2016; OLAI L, et al., 2015; CAMAK DJ, 2015; OLIVIER CL, et al., 2017).

Em contrapartida, no estudo de Costa TF, et al. (2016), 51,47\% dos cuidadores de sua amostra eram filhos, esta característica também foi encontrada em outros estudos (WU MH, et al., 2015; KRIEGER T, et al., 2016; SILVA JK, et al., 2016). De acordo com Torbica A, et al (2015), os filhos assumem o cuidado como uma obrigação moral e retribuição do cuidado e afeto; sendo geralmente assumido pela filha. Neste contexto, os autores ressaltam alguns impactos na vida deste cuidador como a dificuldade de conciliar este novo papel assumido com os seus deveres acadêmicos, profissionais e sociais trazendo também prejuízos na contribuição para a previdência social.

Em estudos de Caro CC, et al (2018), Olai L, et al (2015) e Haley WE, et al (2015), a amostra de cuidadores incluía predominantemente casados ou um relacionamento estável e com baixos níveis de escolaridade. Silva $\mathrm{JK}$, et al (2016), concluiu que $69,2 \%$ dos cuidadores possuíam tempo de estudo inferior a nove anos; resultado análogo a este foi constatado por Caro CC, et al (2018) e Costa TF et al (2016).

Porém, no estudo de Wu MH et al (2015), elaborado na Alemanha, 78\% dos cuidadores tinham nível educacional alto. A partir disso, Camicia M e Lutz BJ (2016) e Zhang L, et al (2019) destaca que o cuidador deve ter conhecimento sobre a condição e o plano de cuidados do paciente, devendo ser avaliado quanto à sua capacidade de prestar os cuidados necessários e sua prontidão para assumir o papel de cuidador domiciliar do paciente.

A média de idade dos cuidadores foi superior a 55 anos em vários estudos (PAI HC e TSAY YC, 2016; KUO YW, et al., 2016; SILVA JK, et al., 2016; MCLENNON SM, et al., 2014). Já no estudo de Olai L, et al. (2015) a idade média dos cuidadores pesquisados era de 63 anos, sendo ressaltado por esses autores que pessoas em processo de senescência estão cuidando de idosos já que o AVC atinge mais comumente essa faixa etária. Quanto à situação profissional, houve predominância de cuidadores desempregados e baixa renda salarial (COSTA TF, et al., 2016; CARO CC, et al., 2018; SILVA JK, et al., 2016). Em um estudo realizado na Espanha por Espuela LF, et al (2015), a renda familiar da maioria dos cuidadores variava entre 500 a 1000 euros (equivalendo cerca de $R \$ 1596,00$ a 3.193,00 neste período).

Em contrapartida, um estudo brasileiro realizado por Silva JK, et al (2016), evidenciou-se que a renda mensal familiar de $84,6 \%$ dos cuidadores correspondia cerca de $R \$ 233,00$ a 2.108,00 com média de $R \$$ 852,10; além disso, foi constatado que não possuíam qualquer remuneração salarial, sendo o dinheiro recebido proveniente de benefícios próprios de aposentadorias. Resultado semelhante a este foi encontrado no estudo de Costa TF, et al (2016), também realizado no Brasil.

Devido a dedicação assistencial ao familiar, a renda e a situação econômica dos cuidadores se encontra dependente, pois a carga horária extensa impossibilita os cuidadores de manterem seus empregos ou se dedicarem à procura de um trabalho como fonte de renda extra. Dessa forma, a aposentadoria do familiar tem sido a principal renda financeira usada para atender suas necessidades básicas, mesmo que de maneira precária (SILVA JK, et al., 2016).

No entanto, Caro CC, et al. (2018) aponta outras possíveis razões para redução na renda dos cuidadores como a preferência por trabalhos informais temporários, pois permitem disponibilidade para se dedicar ao familiar enfermo; ademais, foi descrito que a renda dos cuidadores de pacientes com AVC diminui após a alta hospitalar de seu familiar, pois há uma despesa adicional de assistência médica após um AVC, impondo um ônus financeiro. 
Estudos demonstram que os cuidadores se dedicam em média 20 horas por dia ao cuidado de seus familiares sobreviventes ao AVC, sendo que a demanda por assistência domiciliar varia de acordo com o grau de incapacidade física (SILVA JK, et al., 2016; ESPUELA LF, et al., 2015). Assim, há a necessidade que os cuidadores e o doente com incapacidades residam juntos (CARO CC, et al 2018; OLAI L, et al 2015).

Diante dos resultados identificados, faz-se necessário a profunda compreensão da complexidade de cuidados que o cuidador informal necessita aplicar ao paciente pós AVC em seu retorno ao domicílio. Sendo assim, frente a necessidade de oferecer um material científico informativo e educativo direcionado a essa população, a Associação Brasil AVC (ABAVC) (2019) desenvolveu um caderno apresentando a demanda de atenção em saúde que o paciente necessitará em ambiente domiciliar, dentre a gama de cuidados, salientase: atividades de autocuidado, que comumente ficarão prejudicadas; e, portanto, é fundamental que o cuidador tenha orientação sobre como estimular esse autocuidado e promover os cuidados de higiene até que o mesmo alcance autonomia.

De acordo com Krieger T, et al (2016), o apoio ao cuidador deve envolver toda a trajetória de cuidados ao paciente com AVC em fase de reabilitação. Dessa maneira, é indispensável que este cuidador seja bem orientado na alta hospitalar pelos profissionais de saúde e nesse aspecto o enfermeiro tem papel fundamental, pois tem papel de educador, orientando os cuidados destinados a prevenção de riscos de infecção, sendo enfaticamente apresentado a ele a importância da frequente higienização das mãos e dos cuidados com dispositivos como sonda nasoenteral ou gastrostomia (CARO CC, et al., 2018).

Um importante cuidado empregado pelo cuidador informal, será a administração de medicamentos, o mesmo deverá ser bem instruído quanto as diversas formas de administração, clareza quanto ao conteúdo da prescrição médica, horário, dose e estar atento para renovação das receitas para que não haja interrupção no tratamento medicamentoso (ARRUDA GO, et al., 2018).

É válido ressaltar também sobre as prevenções de lesões por pressão, considerando que muitos pacientes passarão por longos períodos com restrição de movimentação, gerando assim a necessidade de orientação ao cuidador informal quanto a proteção da pele, especialmente em áreas de proeminências ósseas com o uso de coxins, almofadas e travesseiros, bem como a mudança de decúbito, oferecendo conforto e bem estar ao paciente, e consequentemente ao cuidador por saber o que fazer e se sentir mais forte e resolutivo nas ações. O risco de queda também deve ser valorizado pelo olhar dos cuidadores, devendo ser advertido quanto às limitações motoras atuais do paciente e condições ambientais como piso escorregadio, tapetes, calçados inadequados e iluminação insuficiente (CAMAK DJ, 2015).

Exercícios de fisioterapia devem ser empregados precocemente após o evento neurológico, pois o comprometimento motor é uma condição comum após o estabelecimento do AVC, principalmente lesões de ombro; dessa forma, mesmo que haja a assistência de um profissional fisioterapeuta, o qual se faz indispensável durante a reabilitação, outros exercícios devem ser orientados para que ocorra o estímulo muscular contínuo em sua residência (CAMAK DJ, 2015; ARRUDA GO, et al., 2018).

Mudanças na fala e linguagem, são condições também comuns após o AVC, assim sendo, é indispensável orientações quanto aos cuidados com a afasia, uma vez que o avanço de um paciente afásico depende de múltiplos fatores, principalmente de auxílio e apoio das pessoas que o circundam; ademais, destaca-se a disfagia, condição em que afeta diretamente a decisão do tipo de nutrição que será ofertada a este paciente (ESPUELA LF, et al. 2015; OLAI L et al., 2015).

Segundo Camak DJ (2015) a nutrição, por sua vez, deve ser explanada ao cuidador de forma que a orientação sobre esta temática vá além da forma e conteúdo, haja em vista sua interação com os anticoagulantes orais e alguns alimentos consumidos; visando assim, o restabelecimento da condição física de saúde através de uma alimentação saudável.

Portanto, diante da complexa demanda de cuidados inseridos neste cenário, cresce a sensação de isolamento do cuidador devido ao aumento das obrigações de prestação de cuidados; logo, é fundamental que os profissionais de saúde avaliem a sobrecarga do cuidador e trace intervenções para conciliar o cuidado às suas outras atividades cotidianas e identifique serviços de suporte comunitário (CARO CC, et al., 2018; KRIEGER T, et al., 2016). 
Dar suporte aos cuidadores por meio de uma equipe multidisciplinar com ênfase ao enfermeiro é de extrema importância, pois com tantas demandas é necessário que os cuidadores sintam-se preparados para o desenvolvimento dos cuidados a fim de garantir qualidade de vida às pessoas acometidas por AVC e consequentemente a qualidade de vida aos cuidadores (MARTINS e SANTOS, 2020; SANTOS NO, et al., 2020).

\section{CONSIDERAÇÕES FINAIS}

Essa abordagem ampliou o conhecimento acerca do perfil do cuidador informal de pacientes com sequelas pós AVC. Assim, pode-se constatar que a mulher é a principal cuidadora; o cuidado tem sido assumido principalmente por cônjuges ou filhas. Houve predominância de cuidadores casados. Ademais, foi evidenciado baixos níveis de escolaridade entre os cuidadores. A média de idade dos cuidadores informais foi de 55 anos de idade. Além disso, a maioria dos cuidadores encontram-se em situação de desemprego e se dedicam em média 20 horas por dia ao cuidado de seus familiares. O conhecimento desse perfil pode orientar os profissionais de saúde a traçar um plano de cuidados melhor direcionado ao cuidador informal.

\section{REFERÊNCIAS}

1. ARAUJO JB, et al. Sobrecarga de cuidadores familiares e independência funcional de pacientes pós-acidente vascular encefálico. Rev. Ciênc. Méd., 2016; 25(3): 107-113.

2. ARRUDA GO, et al. Percepções da família acerca do cuidado ao homem com alguma condição crônica. Ciência Cuidado e Saúde, 2018; 17(1):1-10.

3. BOCCHI, SC. Vivenciando a sobrecarga ao vir-a-ser um cuidador familiar de pessoa com acidente vascular cerebral (AVC): análise do conhecimento. Rev. Latino-Am. Enfermagem, 2004; 12(1): 115-121.

4. Brasil, Ministério da saúde. Linha de Cuidados em Acidente Vascular Cerebral (AVC) na Rede de Atenção às Urgências e emergências. Brasília, 2012.

5. CAMAK DJ. Addressing the burden of stroke caregivers: a literature review. Journal of Clinical Nursing, 2015; 17(18): 23762382.

6. CAMICIA M, LUTZ, BJ. Nursing's Role in Successful Transitions Across Settings. Stroke, 2016; 47(11): 246-249.

7. CARO CC, et al. Burden and Quality of Life of Family Caregivers of Stroke Patients. Occupational Therapy In Health Care, 2018; 32(2): 154-171.

8. CARO CC, et al. Independence and cognition post-stroke and its relationship to burden and quality of life of family caregivers. Topics in Stroke Rehabilitation, 2016; 24(3): 1-6.

9. COSTA TF, et al. Acidente vascular encefálico: características do paciente e qualidade de vida de cuidadores. Rev. Bras. Enfermagem, 2016; 69(5): 933-939.

10. ESPUELA LF, et al. Critical points in the experience of spouse caregivers of patients who have suffered a stroke - a phenomenological interpretive study. PLOS ONE, 2018; 13(4):1-16.

11. ESPUELA LF, et al. Impacto en la calidad de vida en cuidadores de supervivientes de un ictus. Enfermería Clinica, 2015; 25(2):49-56

12. HALEY WE. Long-term impact of stroke on family caregiver well-being. American Academy of Neurology, 2015; 84(13): 13231329.

13. HEBEL K, et al. Health education for stroke patient carers: Does it affect functional status improvement in patients after ischemic stroke? Applied Nursing Research, 2014; 27(3): 7-12.

14. KUO, YW. A home-based training programme improves family caregivers' oral care practices with stroke survivors: a randomized controlled trial. Int. J. Dent. Hygiene, 2015; 14(2): 82-91.

15. MARTINS R, SANTOS C. Capacitação do cuidador informal: o papel dos enfermeiros no processo de gestão da doença. Rev. Gestão e Desenvolvimento, 2020; 28: 117-137.

16. MCLENNON, SM. Task Difficulty and Life Changes among Stroke Family Caregivers: Relationship to Depressive Symptoms. Arch. Phys. Med. Rehabilitation, 2014; 95(12): 2484-2490.

17. OLAI L, et al. Life situations and the care burden for stroke patients and their informal caregivers in a prospective cohort study. Ups. J. Med. Sciences, 2015; 120(4): 290-298.

18. OLIVIER CL, et al. To be or not to be? A caregiver's question: the lived experience of a stroke family during the first 18 months poststroke. Scand. J. Caring Sci, 2017; 32(1): 1-10.

19. OPAS - Organização Pan-Americana da Saúde. As 10 principais causas de mortes no mundo. Brasília (DF); 2018.

20. PAI HC, TSAI YC. The Effect of Cognitive Appraisal on Quality of Life of Providers of Home Care for Patients With Stroke. Journal of Neuroscience Nursing, 2016; 48(1): 2-11.

21. SANTOS NO, et al. Construção e validação de protocolo assistencial de enfermagem com intervenções educativas para cuidadores familiares de idosos após Acidente Vascular Cerebral. Rev. Brasileira de Enfermagem, 2020; 73(3): 1-9.

22. SILVA JK, et al. Perfil de cuidadores familiares de idosos após o Acidente Vascular Cerebral. Rev. Enferm. UFPE, 2016; 10(10): 3727-3733.

23. TORBICA A, et al. Does informal care impact utilization of healthcare services? Evidence from a longitudinal study of stroke patients. Social Science \& Medicine, 2015; 124(1): 29-38.

24. WU, MH, et al. The effect of cognitive appraisal in middle-aged women stroke survivors and the psychological health of their caregivers: a follow-up study. J. Clin. Nursing, 2015; 24(21-22): 3155-3164.

25. ZHANG L, et al. A newly designed intensive caregiver education program reduces cognitive impairment, anxiety, and depression in patients with acute ischemic stroke. Braz J Med Biol Res, 2019; 52(9): 8533-8546. 